\title{
The use of $3 \mathrm{~d}$ scanner for testing changes in shape of human limbs under the influence of external mechanical load
}

\author{
Kamila Kasperska ${ }^{1}$, Michał Wieczorowski ${ }^{1, *}$, and Jolanta B. Krolczyk ${ }^{2}$ \\ ${ }^{1}$ Poznan University of Technology, Faculty of Mechanical Engineering and Management, 3 Piotrowo Street, 60-965 Poznan, Poland \\ ${ }^{2}$ Opole University of Technology, Faculty of Mechanical Engineering, 76 Proszkowska Street, 45-758 Opole, Poland
}

\begin{abstract}
Three-dimensional scanning is used in many fields: medicine, architecture, industry, reverse engineering. The aim of the article was to analyze the changes in the shape of the limbs under the influence of a mechanical external load using the method of three-dimensional scanner uses white light technology. The paper presents a system of human movement, passive part - skeleton and active part - the muscles, and principles of their interaction, which results in a change of the position of the body. Furthermore, by using the 3D scan, the differences in appearance of the arm and leg depending on the size of the external load in different positions have been presented. The paper shows that with increasing load, which muscles must prevent, increases the volume of certain parts of the legs, while another parts of them will be reduced. Results of the research using three-dimensional scanner allow determining what impact on changing the legs shape has an external mechanical load.
\end{abstract}

\section{Introduction}

3D scanning is becoming increasingly popular as a measuring tool [1]. It makes it possible to check the quality of the produced elements by comparing the 3D mesh of the product with the Computer Aided Design (CAD) model. It provides information on the areas where the differences are bigger which leads to the improvement in the technological process and elimination of the causes of the resulting errors. It may be applied to many areas: medicine, architecture, industry, reverse engineering. 3D metrology has been known also for many years as an inspection method [25]. 3D scanning has become also a tool used in almost every area of life. People are constantly striving to improve the imaging tools in order to obtain the results that are almost identical to the real objects and the preparation of the test surface was unnecessary. The 3D Go!Scan scanner used in the research is one of the latest solutions and the image is collected and set based on the colour and texture.

The optical method, lighting with structural light has a number of advantages, i.e.: precision of measurement by an order better than in the case of tracing, non-contact measurement, possibility of automating the measurement [6-7].

Automation progress is related to computer aided manufacturing. CAD is one of the kinds of computer aided production. It is often combined with Computer Aided Manufacturing in order to obtain fluent transition from the stage of design to the stage of production [8]. Computer Integrated Manufacturing (CIM) is a synthesis of the two previous kinds [9-11].
The aim of the article was to analyze the changes in the shape of the limbs under the influence of a mechanical external load using the method of threedimensional scanner uses white light technology.

\section{Materials and methods}

The research part included the determination of the differences in the appearance of the arm and lower leg depending on the size of the external load which they were subjected to in the given position. It is assumed that when the load which the muscles need to counteract is increased, the volume of some parts of limbs will increase and other parts will be reduced.

The manual 3D Creaform scanner using the technology of white light was used for the research. The scanner takes a quick measurement of spatial objects with complex geometry. The advantage is that the tested object must not be mounted rigidly, its small movements are allowable. The device is up to $0.100 \mathrm{~mm}$ accurate.

The objective of the research part was to examine how the load affects the external shape of the limb muscles. Assumptions for the research in the field of weight training:

$>$ exercise, which will cause the change of shape of the given part of the limb and it will be possible to illustrate its entire circumference,

$>$ time to stand still in the given position should range between 45 and $60 \mathrm{~s}$,

$>$ test surface should be smooth; removal of body hair is necessary,

$>$ use of available sports equipment, namely assembled

\footnotetext{
Corresponding author: michal.wieczorowski@put.poznan.pl
} 
dumb-bells.

Two parts of the body were used in the research: a calf and an arm.

- for a calf: calf raises with dumb-bells - the threeheaded muscle of the calf becomes visible,

- for an arm: dumbbell arm curls or pull-downs, standing position - mainly the biceps brachii works.

Table 1. Load plan for individual persons.

\begin{tabular}{|l|l|}
\hline & Arm load - biceps brachii \\
\hline \multirow{4}{*}{ Subject No. 1 } & no load \\
\cline { 2 - 2 } & $3 \mathrm{~kg}$ \\
\cline { 2 - 2 } & $5 \mathrm{~kg}$ \\
\cline { 2 - 2 } & $13 \mathrm{~kg}$ \\
\hline & Arm load - biceps brachii \\
\hline \multirow{4}{*}{ Subject No. 2 } & no load \\
\cline { 2 - 2 } & $3 \mathrm{~kg}$ \\
\cline { 2 - 2 } & $5 \mathrm{~kg}$ \\
\cline { 2 - 2 } & $13 \mathrm{~kg}$ \\
\hline \multirow{5}{*}{ Subject No. 2 } & Lower leg - calf \\
\cline { 2 - 2 } & natural position, standing \\
\cline { 2 - 3 } & muscle flexed, no load \\
\cline { 2 - 2 } & muscle flexed, 11 kg \\
\hline
\end{tabular}

Two people took part in the tests: subject No. 1: a man who attends the gym for pleasure and subject No. 2: a woman who professionally trains powerlifting. In theory, there was no need for special preparation of the test surface but in order to shorten the time of model creation it was decided to tape the part of the limbs with markers which help to find the correct location of the newly obtained data. In the case of the upper limb it turned out to be sufficient but there was a problem with the lower limb. It was a big surprise, because the geometry of lower limb is far less complex than the geometry of arm and it seemed to be an easier object to scan with a 3D scanner. Additional, colourful patterns were drawn, apart from the points stuck on the limb, in order to facilitate the operation of the device. Two models were scanned for different loads, which are presented in Tabela 1, and adjusted to the capabilities of the research participants. The largest weight constituted about $60 \%$ of the first maximum.

The results of each measurement were acquired in the form of a cloud of points representing the part of the limb when doing a specific exercise flexing the working parts of the muscles. Most of the programs that have the function of editing the point clouds represent it omitting the actual colours. The scanner that was used creates a three-dimensional model based on colour and texture of the object, therefore, the ViewShape program was used to show the results as per Fig. 1 and 2.

Editing the measurement data was carried out in the GOM Inspect program. It is used primarily for analysis of information obtained during the 3D imaging during the quality control, product preparation and production.

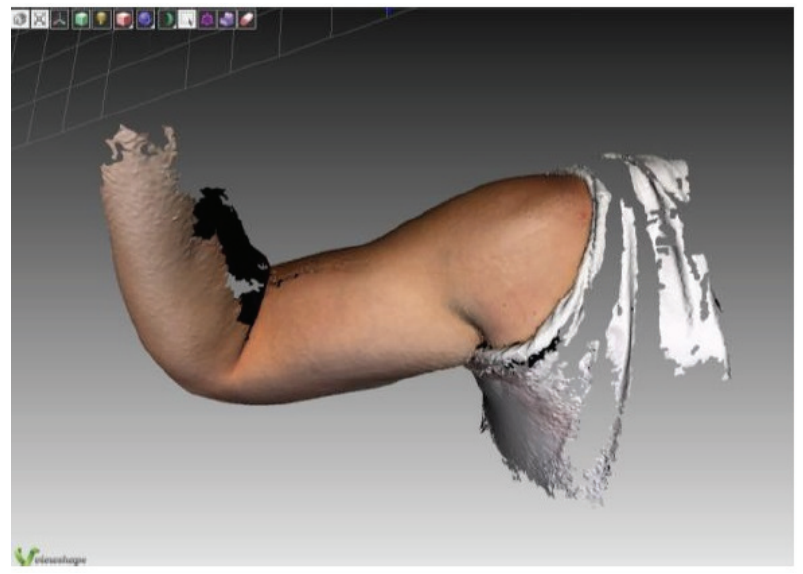

Fig. 1. Obtained point clouds presenting the examined parts of the body in the ViewShape program: arm.

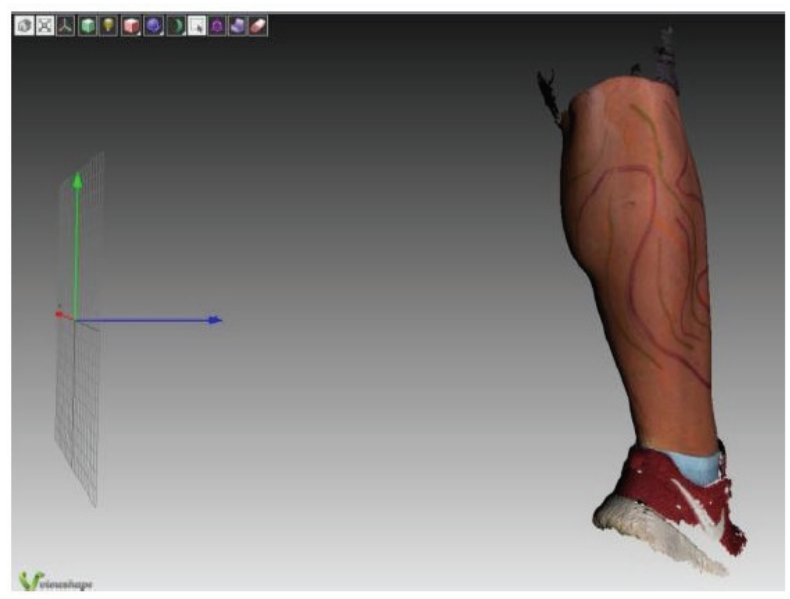

Fig. 2. Obtained point clouds presenting the examined parts of the body in the ViewShape program: lower leg.

\section{Results}

The main task to be carried out in the research part was the determination of the influence of the lifted weight on the appearance of the limbs. In order to do this they were scanned with the use of 3D method, which made it possible to determine the length of the cross sections of the examined parts in the significant areas, that is in the areas where, in theory, the biggest changes should occur. We use the GOM Inspect program to examine the circumferences, especially its function of determining the cross section in a specific area and then automatic generating of its length. Determining the circumference of the given part of the body using the GOM Inspect V8 SR2 program is shown in Fig. 3-4.

In Fig. 5 the changing shape of the arm (biceps) is shown - a 3D image - when doing exercises without load and a $3 \mathrm{D}$ image of the arm when doing exercises with the load of $3 \mathrm{~kg}$ (Fig. 6).

The exercise performed is characterised by the most intensive work of the biceps brachii, therefore, mainly the fact that this muscle is convex will have an influence on changing the shape of the upper limb.

Table 2 presents the results of the research. 


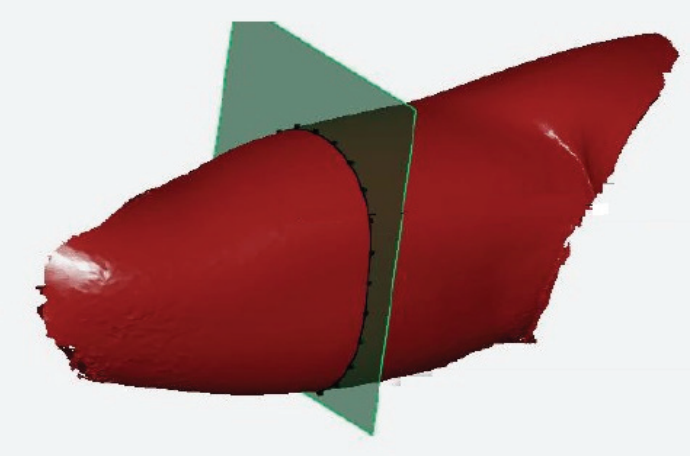

Fig. 3. Determining of the area of the cross section.

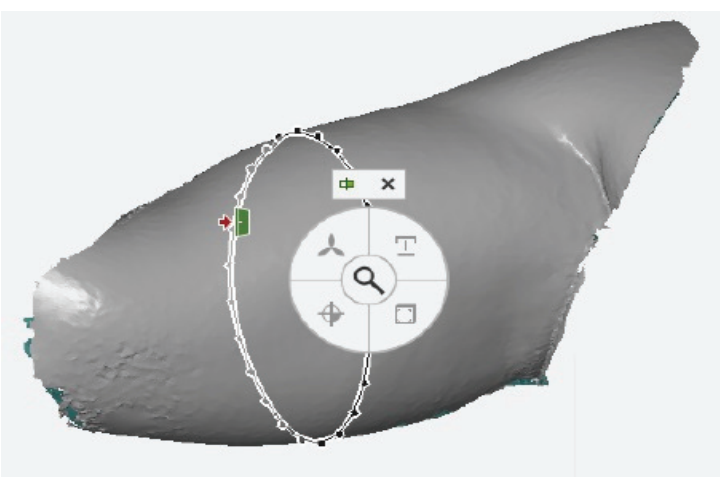

Fig. 4. Measuring the length of the cross section circumference.

Table 2. The arm circumference when exercising with different loads.

\begin{tabular}{|l|l|}
\hline \multicolumn{2}{|c|}{ Subject No. 1 } \\
\hline Load [kg] & Circumference [mm] \\
\hline no load & 386.2 \\
\hline 3 & 389.1 \\
\hline 5 & 392.5 \\
\hline 13 & unsuccessful \\
\hline \multicolumn{2}{|c|}{ Subject No. 2 } \\
\hline Load [kg] & Circumference [mm] \\
\hline no load & 386.7 \\
\hline 3 & 388.1 \\
\hline 5 & 389.9 \\
\hline 13 & 393.4 \\
\hline
\end{tabular}

A similar operation was carried out to observe changes of the calf shape when standing and performing the calf raise exercise with a load of $11 \mathrm{~kg}$. Calf raises engage the muscles of the calf, namely the soleus and inner and outer head of the gastrocnemius muscle. A significant change of the shape during the exercise is noticed, even without load. Tab. 3 presents the results of the research.

Table 3. The length of the calf circumference in different positions for subject no 2 .

\begin{tabular}{|l|l|}
\hline Load $[\mathrm{kg}]$ & Circumference [mm] \\
\hline Standing position & 431.4 \\
\hline Calf rises & 440.5 \\
\hline Calf rises + load of $11 \mathrm{~kg}$ & 441.2 \\
\hline
\end{tabular}

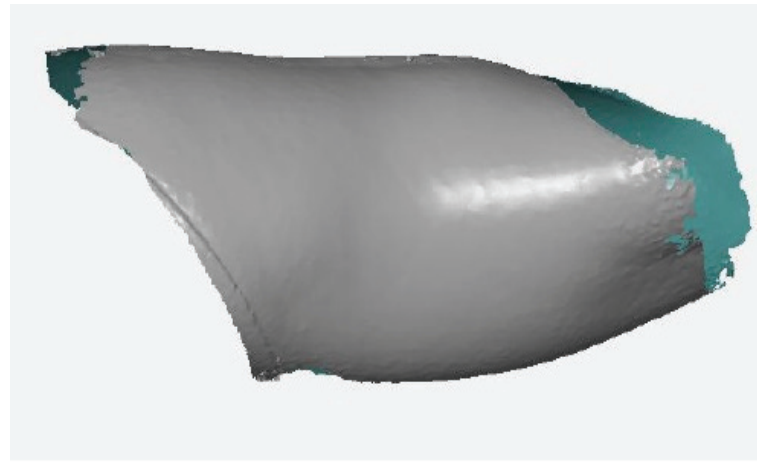

Fig. 5. Change of the arm shape when exercising without load.

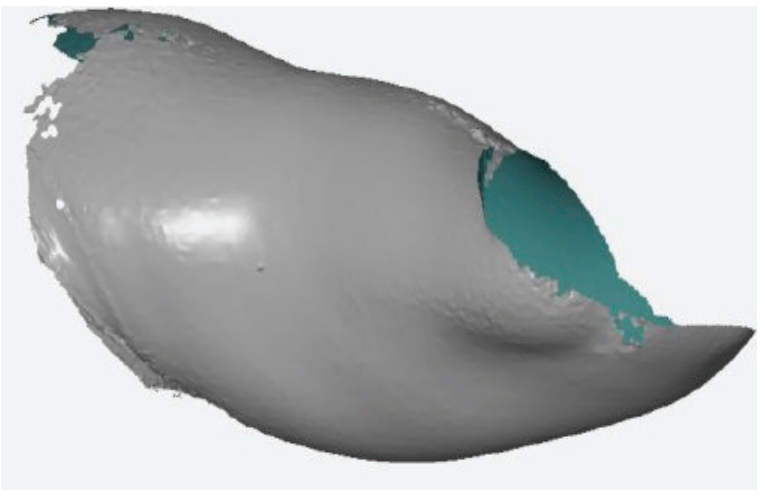

Fig. 6. Change of the arm shape when exercising with a load of $3 \mathrm{~kg}$.

Apart from the examination of the changes in limb circumference during the movement without load or with a load, also the specification of their transformations was considered. The option of the Geomagic Control was used here. It makes it possible to fit two different 3D meshes in the STL format with the use of "Best Fit Alignment" method and then to compare them by means of 3D method and to create colourful maps of deviations. It shows the parts of the limb in which the modifications were the largest as well as the level on which they were located. Due to the fact that each of the muscles works differently during the particular movement, there are surpluses in some areas and losses in other areas.

In Fig. 7 and 8 the selected research results are presented. It was observed for the map shown in Fig. 7 that the biceps brachii muscle became much more visible in the front view and the triceps brachii muscle became slightly more visible in the back view. The shape change in the area of the larger muscles caused cracks in the lower and upper sections of the limb.

Results in the form of colourful map of deviations for the lower leg when standing and during calf raises shown in Fig. 8.

Analysing the obtained results it may be concluded that the flexion of the three-headed calf muscle caused the cracks on the outer side along the tested muscle and on the inner side under the muscle as well as a slight increase in its volume. 

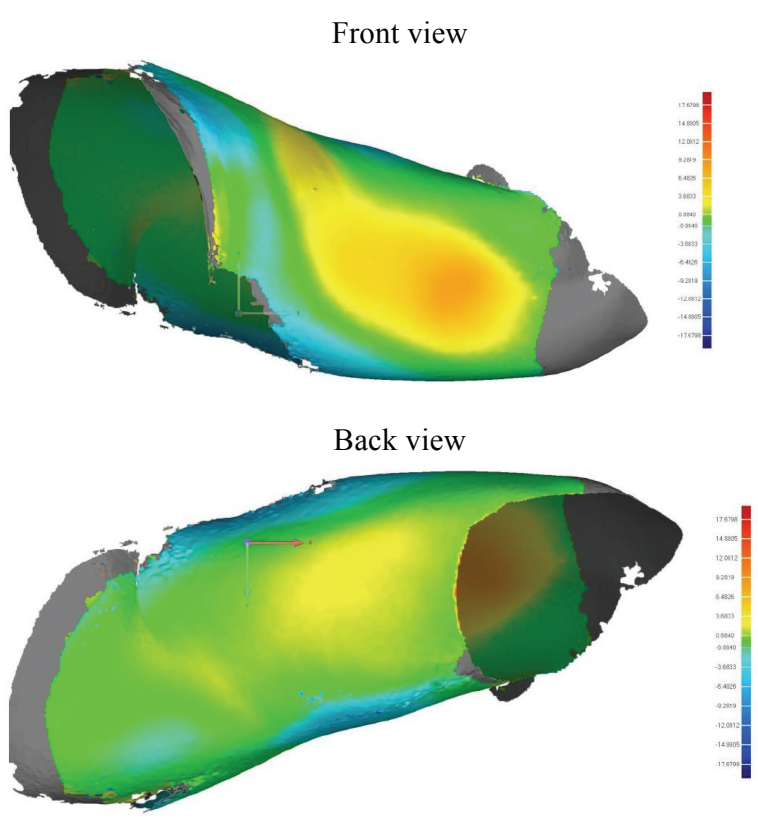

Fig. 7. Map of deviations for subject No. 1 comparing the data arising during the performance of the arm exercises without load and with the load of $3 \mathrm{~kg}$.

\section{Conclusions}

1. The results obtained thanks to the research performed make it possible to determine the influence of the external mechanical load on the shape change of the limb. It may be noted that in no case two identical 3D meshes were obtained.

2. The first step was the determination of the length of the limb circumference in the relevant area, that is in the area where the volume change was the most significant. It made it possible to advance a thesis assuming that when the external load influencing the arm and lower leg is increased, the tested length also increases. This process is influenced by many factors which will be described later.

3. Determination of the areas where the changes were the most significant was possible thanks to the creation of the colourful maps of deviations, which identified the areas of modifications. It enabled us also to realize that the muscle flexion may lead to the increased volume in one area but also to the losses of volume in another area.

4. In the case of upper limb the biceps branchii muscle is slightly more convex for both subjects, with a small increase in the volume of the triceps branchii muscle at the same time. The flexion of these muscle areas during the exercise performance made the cracks in the upper and lower area of the arm more visible.

5. This was not the case for the lower leg. The first measurement was taken when the subject was in the standing position with both feet on the ground and the other during the calf raise without weights. It made it possible to show how the lack of muscle flexion influences the shape of the calf. A slight increase in its volume was also noted, with
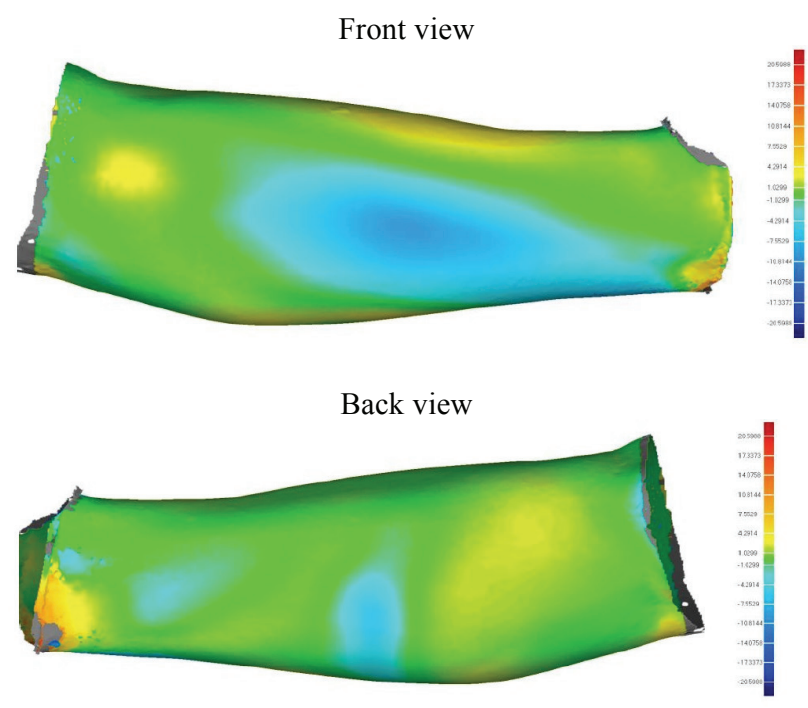

Fig. 8. Map of deviations for subject No. 2 for the lower leg when standing and during the calf raise.

significant losses on the edges of the parts which conduct the greatest work during the performed activity. In this case, the additional load only made the changes that appeared before more visible.

\section{References}

1. T.G. Mathia, P. Pawlus, M. Wieczorowski, Wear 271 (2011)

2. P. Nieslony, G.M. Krolczyk, K. Zak, R.W. Maruda, S. Legutko, Precis Eng. 47 (2017)

3. G.M. Krolczyk, J.B. Krolczyk, R.W. Maruda, S. Legutko, M. Tomaszewski, Measurement $\mathbf{8 8}$ (2016)

4. C. Zhang, Z. Li, C. Hu, S. Chen, J. Wang, X. Zhang, Meas. Sci .Technol.. 28 (2017)

5. J.B. Krolczyk, B. Gapinski, G.M. Krolczyk, I. Samardzic, R.W. Maruda, K. Soucek, Y. Javadi, S. Legutko, P. Nieslony, L. Stas, The. Vjesn. 23 (1), (2016)

6. B. Curless, Overview of active vision techniques (SIGGRAPH 99 Course on 3D Photography, 1999)

7. J. Geng, Structured-light 3D surface imaging: a tutorial (Optical Society of America, 2011)

8. S. Wojciechowski, P. Twardowski, M. Pelic, Procedia CIRP 14, 113-118 (2014)

9. S. Wojciechowski, R.W. Maruda, P. Nieslony, G.M. Krolczyk, Int. J. Mech. Sci. 119, 360-369 (2016)

10. S. Wojciechowski, P. Twardowski, M. Pelic, P.W. Maruda, S. Barrans, S., G.M. Krolczyk, Precis Eng. 46, 158-165 (2016)

11. S. Jun, O. Kochan, Meas. Sci. Rev. 14 (1), 29-34 (2014) 